\section{The road to silence}

In the eukaryotic nucleus, accessible euchromatin facilitates transcriptional activity whereas compact heterochromatin maintains transcriptional silence. And, although these opposing chromatin states are intrinsically stable, transitions between euchromatin and heterochromatin are necessary to accommodate changes in gene expression.

Now, reporting in The EMBO Journal, Yael Katan-Khaykovich and Kevin Struhl show that the conversion of euchromatin to heterochromatin is promoted by stepwise changes in histone modifications. Surprisingly, the formation of heterochromatin is a gradual process that requires several rounds of cell division, and the transition to silent chromatin is marked by intermediate chromatin states in which transcriptional repression is incomplete.

To determine the temporal kinetics of heterochromatin formation, Khaykovich and Struhl focused on the $H M R$ silent mating type loci and a subtelomeric region of the Saccharomyces cerevisiae genome. Heterochromatin formation can be induced at these sites by the overexpression of the silencing protein Sir3, which allows a time-course analysis of the histone modifications that accompany the assembly of silent chromatin.

Prior to Sir3 overexpression, the target loci bear the marks of 'active chromatin' - acetylated histone 4 (H4)-K16, methylated H3-K4 and methylated H3-K79. Shortly after induction of heterochromatin formation, during the so-called 'initiation phase', there is rapid histone deacetylation, although euchromatic

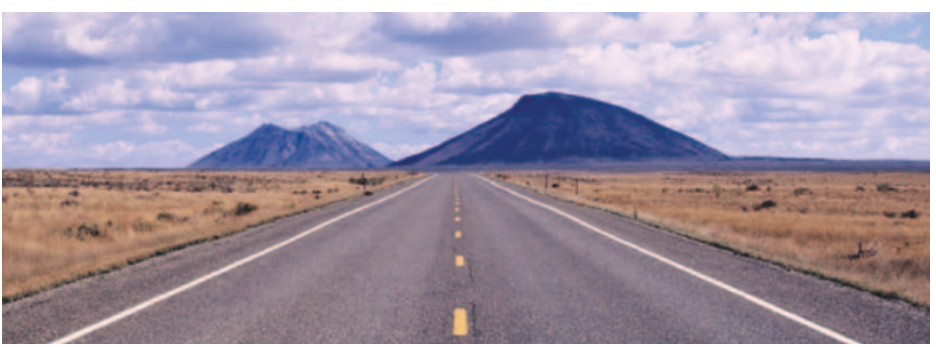

histone methyl marks persist. This 'partial heterochromatin' domain is characterized by suboptimal binding of silencing proteins and by low levels of transcriptional activity. During the subsequent 'maturation phase', which takes place over several cell generations, methylated H3-K4 and methylated H3-K79 are removed by histone exchange that is both DNA-replication dependent and DNA-replication independent. The authors propose that the gradual removal of euchromatic histone methyl marks allows further binding of silencing proteins and ultimately complete transcriptional shut down.

This model embodies the principle that euchromatic histone modifications impede the formation of heterochromatin, and studies using mutant $S$. cerevisiae strains confirmed this notion. For example, strains that lacked Dot1, the H3-K79 methylase, accumulated silencing proteins rapidly at the $H M R$, whereas the loss of the histone acetylase Sas2 resulted in the rapid spread of heterochromatin away from the telomere.

Although S. cerevisiae notably lacks H3-K9 methylation - the trademark of constitutive heterochromatin in higher eukaryotes - the conservation of other heterochromatin components indicates that silencing pathways will be characterized by similar landmarks across the species.

Shannon Amoils

\section{6) References and links} ORIGINAL RESEARCH PAPER Katan-

Khaykovich, Y. \& Struhl, K. Heterochromatin formation involves changes in histone modifications over multiple cell generations. EMBO J. 24, 2138-2149 (2005)
Physical association and coordinate function of the H3 K4 methyltransferase MLL1 and the H4 K16 acetyltransferase MOF.

Dou, Y. et al. Cell 121, 873-885 (2005)

Acetylation of histone 4 (H4) on K16 and methylation of K4 on $\mathrm{H} 3$ are independently associated with transcriptional activation, but evidence indicates that these two modifications often function in coordinated combinations. Dou et al. have now discovered that the $\mathrm{H} 3 \mathrm{~K} 4$ methyltransferase MLL1 is part of a complex that contains, among other components, MOF, which specifically acetylates $\mathrm{H} 4 \mathrm{~K} 16$. The histone methyltransferase and histone acetyltransferase activities of the complex were required for transcriptional activation on a chromatin template and an MLL1-target gene in vivo. So, the isolation of this complex provides a functional connection between the two histone modifications.

\section{CELL FATE}

Delta-Notch signalling controls commitment to a secretory fate in the zebrafish intestine.

Crosnier, C. et al. Development 132, 1093-1104 (2005)

Notch $\gamma$-secretase inhibition turns proliferative cells in intestinal crypts and adenomas into goblet cells.

van Es, J. H. et al. Nature 435, 959-963 (2005)

Notch signals control the fate of immature progenitor cells in the intestine.

Fre, S. et al. Nature 435, 964-968 (2005)

The first of these three papers reports an increase in the number of secretory cells at the expense of absorptive cells in the zebrafish gut in the absence of Delta-Notch signalling. The authors of the second and third papers also studied the intestine, but in mice. When van Es et al. blocked Delta-Notch signalling, this caused proliferative cells in the crypt to convert into differentiated goblet cells. Conversely, Fre et al. activated Notch signalling, which prevented secretory-cell differentiation and increased the population of progenitor cells.

\section{ENDOCYTOSIS}

Protein kinase activity of phosphoinositide 3 -kinase regulates $\beta$-adrenergic receptor endocytosis.

Naga Prasad, S. V. et al. Nature Cell Biol. 10 July 2005 (doi:10.1038/ncb1278)

These authors have identified the first protein target of phosphoinositide 3-kinase (PI3K) - a protein and lipid kinase, the lipid-kinase activity of which is known to be important in signalling. They have found that the cytoskeletal protein non-muscle tropomyosin is a substrate for the protein-kinase activity of PI3K, and that the PI3K-mediated phosphorylation of tropomyosin on Ser61 is essential for the agonist-dependent internalization of the $\beta$-adrenergic receptor. 